\title{
Digitale Transformation im Maschinen- und Anlagenbau. Digitalisierungsstrategien und Gestaltung von Arbeit 4.0
}

\author{
Jürgen Dispan ${ }^{(凶)}$ \\ IMU Institut, Hasenbergstraße 49, 70176 Stuttgart, Deutschland \\ jdispaneimu-institut.de
}

\begin{abstract}
Zusammenfassung. Digitalisierung und Industrie 4.0 spielen im Rahmen der digitalen Vernetzung eine immer größere Rolle für den Maschinenund Anlagenbau - und das sowohl als Anbieter als auch als Anwender digitaler Produkte. Digitale Geschäftsmodelle und neue Wettbewerber aus dem Bereich digitaler Plattformen stellen zunehmend Herausforderungen für die Maschinenbauunternehmen dar. Der Beitrag befasst sich mit den Digitalisierungsstrategien der Maschinenbauunternehmen ebenso wie mit dem Stand der Digitalisierung bei den Prozessen, Produkten und Geschäftsmodellen. Es werden Wirkungen der digitalen Transformation auf Beschäftigung untersucht sowie arbeits- und beschäftigungspolitische Herausforderungen in der Branche aus Sicht von Mitbestimmungsakteuren diskutiert. Daraus werden Gestaltungsfelder und strategische Orientierungen für die Mitbestimmungsträger erarbeitet.
\end{abstract}

Schlüsselwörter: Maschinenbau $\cdot$ Digitalisierung $\cdot$ Beschäftigungswirkungen

\section{Einleitung}

Digitalisierung und Industrie 4.0 sind für den Maschinen- und Anlagenbau sowohl in der Perspektive des Anbieters von digitalisierten Produkten und Services als auch des Anwenders bei den internen Prozessen hochrelevant. So spielen im Rahmen der digitalen Vernetzung zum Beispiel Cyber Physical Systems, Big Data, künstliche Intelligenz, vorausschauende Wartung, digitale Assistenzsysteme und weitere digitale Technologien eine immer größere Rolle für den Maschinenbau. Digitale Geschäftsmodelle und neue Wettbewerber aus der Plattformökonomie stellen zunehmend Herausforderungen für die Maschinenbauunternehmen dar.

Technologische Treiber für die digitale Transformation sind die stark steigenden Rechner- und Speicherleistungen, die neue Formen der künstlichen Intelligenz und ihrer dezentralen Nutzung ermöglichen, die intelligente Sensorik zur gezielten Erfassung großer Datenmengen sowie die zunehmende Vernetzung und weltweite Kommunikation in Echtzeit. Jedoch ist Digitalisierung weit mehr als ein technologischer Wandel. Die Veränderungen sind als Wechselwirkungen zwischen Menschen und Technik, als sozio-technische Systeme zu betrachten. Die erweiterten technischen Möglichkeiten werden erst wirksam, wenn sie von den Menschen in Unternehmen 
und in der Gesellschaft genutzt werden. Erst im Zusammenspiel von Menschen, Technik und Organisation verändert Digitalisierung tatsächlich die Arbeitswelt. Dieses Verständnis von Digitalisierung impliziert, dass die technologische, organisatorische und arbeitsbezogene Dimension eines Wertschöpfungsprozesses gleichermaßen in den Blick genommen wird. Speziell im Maschinenbau kommt zur anwendungsbezogenen Sicht auf Digitalisierung und Industrie 4.0 auch die Sicht als Anbieter von Investitionsgütern für Kunden in vielen Wirtschaftszweigen, die ihrerseits die eigenen Prozesse immer stärker digitalisieren.

Als Kern der deutschen Investitionsgüterindustrie ist der Maschinenbau volkswirtschaftlich und beschäftigungspolitisch überaus bedeutend. Mit ihren weit mehr als einer Million Beschäftigten in 6400 Unternehmen ist die Branche die industrielle Säule Deutschlands. Maschinen und Anlagen stellen eine wichtige Grundlage für die Innovations- und Wettbewerbsfähigkeit der Industrie dar und sie spielen weltweit eine entscheidende Rolle für die Produktivitäts-, Qualitäts- und Kostenentwicklung in produzierenden Unternehmen.

Für die zahlreichen Mitarbeiter in den Unternehmen der Branche ist die digitale Transformation mit Auswirkungen auf Beschäftigungschancen, Arbeitsbedingungen, Kompetenzanforderungen und Qualifikationsbedarfe verbunden. In diesem Kontext befasste sich die Studie „Digitalisierung im Maschinenbau“ mit den branchenspezifischen Herausforderungen durch Digitalisierungsprozesse aus einer arbeitsorientierten Sicht. ${ }^{1}$ Dafür sind erstens Einblicke in die Digitalisierungsstrategien der Maschinenbauunternehmen ebenso wie der Stand der Digitalisierung bei den Produkten und Geschäftsmodellen wie auch bei den internen Prozessen relevant. Auf dieser Grundlage lassen sich im zweiten Schritt Wirkungen und wechselseitige Abhängigkeiten auf die Innovations- und Wettbewerbsfähigkeit der Unternehmen und der Branche sowie auf Beschäftigung und Arbeitspolitik im Maschinenbau analysieren und im dritten Schritt Handlungsbedarfe für die Gestaltung guter Arbeit und für Beteiligung erarbeiten.

Zielsetzung des Forschungsprojekts „Digitalisierung im Maschinenbau“ war es, auf Basis einer fundierten Analyse von Digitalisierungsprozessen in ausgewählten Teilbranchen des Maschinenbaus in Deutschland zum einen Chancen und Risiken für Beschäftigung und Arbeit abzuleiten und zum anderen Gestaltungsfelder für gute Arbeitsbedingungen, sichere Beschäftigungsperspektiven und nachhaltige Personalpolitik in der Branche zu erarbeiten.

Digitalisierung, Industrie 4.0, Arbeit 4.0 und viele weitere 4.0-Themen erleben im wissenschaftlichen Diskurs und in der politischen Debatte eine Hochkonjunktur, die mit einer Vielzahl von Studien, Konferenzen und Publikationen einhergeht.

1 Der vorliegende Beitrag beruht im Wesentlichen auf den Ergebnissen der Studie „Digitalisierung im Maschinenbau“ (Dispan, Schwarz-Kocher 2018), die vom IMU Institut Stuttgart im Auftrag der Hans-Böckler-Stiftung und der IG Metall erstellt wurde. 
Speziell auf den Maschinenbau bezogen gibt es beispielsweise verschiedene Studien und Leitfäden des VDMA (Überblick in VDMA 2019) sowie arbeitswissenschaftliche Untersuchungen wie „Industrie 4.0 - Qualifizierung 2025“ (Pfeiffer et al. 2016), „Digitalisierter Maschinenbau - Wandel und Entwicklungschancen qualifizierter Arbeit“ (Hirsch-Kreinsen 2017) und „Digitalisierung und Arbeit im niedersächsischen Maschinenbau“ (Kuhlmann, Voskamp 2019). ${ }^{2}$

\section{Digitalisierungsstrategien - der Maschinenbau als Anbieter und Anwender}

Inwieweit ist das Thema „Digitalisierung“ in den Strategien von Maschinenbauunternehmen verankert und wie weit sind die Unternehmen auf dem Weg der digitalen Transformation? Entlang von Betriebsfallstudien, Expertengesprächen und Workshops wurde diesen Fragen nachgegangen. Im Ergebnis gibt es in der Breite des heterogenen Maschinenbaus beim Stand der Digitalisierung und bei Digitalisierungsstrategien ein sehr vielfältiges Bild. Viele kleine und mittlere Unternehmen befanden sich zum Stand 2018 erst am Anfang der digitalen Transformation. Bei den untersuchten größeren Unternehmen, die meist zu den Vorreitern bei der digitalen Transformation zählen, wurde die Digitalisierung hingegen strategisch vorangetrieben.

\subsection{Vier Säulen der Digitalisierungsstrategien}

Digitalisierungsstrategien von Maschinenbauunternehmen lassen sich in vier Säulen gliedern, die jeweils unterschiedliche Aspekte von Digitalisierung umfassen und den zwei Feldern externe Angebote (Anbieterperspektive) und interne Prozesse (Anwenderperspektive) zugeordnet werden können (Abb. 1):

1. Erweiterung des eigenen Portfolios um digitalisierte Produkte und Services

2. Entwicklung neuer Geschäftsfelder oder neuer Geschäftsmodelle auf Basis von Künstlicher Intelligenz (KI) und von digitalen Plattformen für das Industrial Internet of Things (IIoT)

3. Vernetzung der Unternehmensprozesse und interne digitale Transformation der Organisation, aber auch überbetriebliche Vernetzung innerhalb des Wertschöpfungsnetzwerks

4. Beteiligung der Beschäftigten und der Betriebsräte sowie Gestaltung der digitalen Transformation durch aktives Change Management und Qualifizierung

2 Vergleiche Dispan, Schwarz-Kocher (2018) für einen ausführlichen Literaturüberblick zur Digitalisierung im Maschinenbau. 


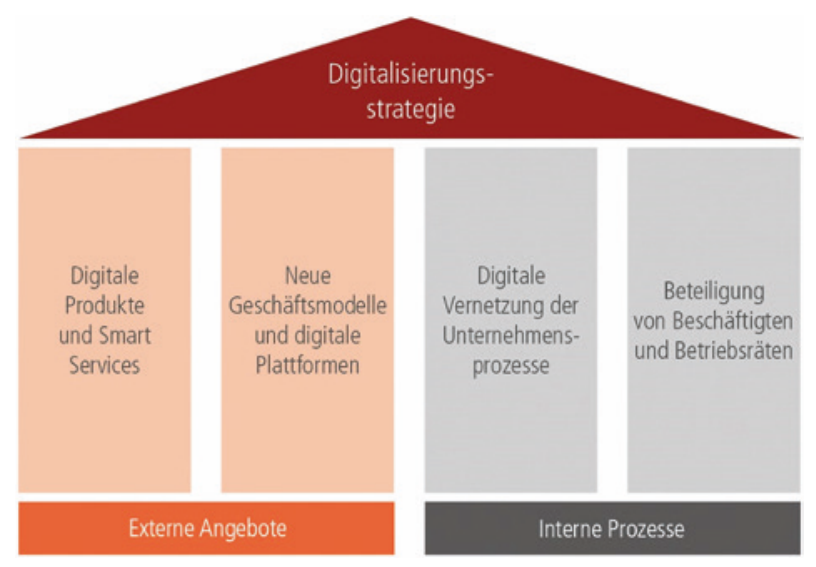

Abb. 1. Vier Säulen der Digitalisierungsstrategien von Maschinenbauunternehmen. (Eigene Darstellung nach IMU Institut)

Mit diesen vier Säulen werden unterschiedliche Perspektiven eingenommen: die des Anbieters und die des Anwenders von digitalen Lösungen. Gerade im Maschinenbau ist Digitalisierung in erster Linie ein stark kundenbezogenes Thema. Es geht darum, mit digitalen Lösungsangeboten zusätzlichen Kundennutzen zu bieten und die Kundenbindung zu erhöhen, wie viele der befragten Experten hervorhoben. Für den Maschinenbau insgesamt ist zu konstatieren, dass die Anbieterseite der Digitalisierung bisher einen deutlich höheren Stellenwert einnimmt als die Anwenderseite mit Industrie 4.0 bei den eigenen Unternehmensprozessen, wo in der Vergangenheit eine schleichende Umsetzung zu beobachten war.

\subsection{Maschinenbau als Anbieter digitaler Lösungen}

Auf die beiden ersten Säulen bezogen hat die Dynamik der digitalen Transformation in den letzten Jahren vor allem bei den größeren Maschinenbauern deutlich an Fahrt gewonnen. Die in betrieblichen Fallstudien Befragten waren sich einig, dass im Maschinenbau ,eine immer stärker werdende Digitalisierungsdynamik“ zu erwarten ist. Die Aufgabe, sich der digitalen Transformation zu stellen, ordnen sie in einem Spektrum von „wichtig“ über ,unumgänglich“ bis „alternativlos“ ein. In puncto Wettbewerbsfähigkeit werde es zu einer „Verschiebung von Kompetenzen bei Stahl und Eisen zu Kompetenzen bei Software und Datenanalyse" kommen. Entsprechend sind digitale Lösungen verstärkt am Markt und es entstehen vermehrt digitale Plattformen aus dem Maschinenbau heraus. So bieten die Unternehmen der Aufzugsbranche mittlerweile digitale Tools für das Monitoring, die vorausschauende Wartung, Störungsmeldungen und die Auswertung von Nutzungsdaten für die Anlagenbetreiber an. In der Fördertechnik werden beispielsweise fahrerlose Transportsysteme, digitales Flottenmanagement und vernetzte Logistiklösungen angeboten. In der Landmaschinentechnik spielen GPS-basierte Assistenzsysteme, autonome Erntemaschinen 
und das Angebot von Smart-Farming-Plattformen eine zunehmende Rolle. Im Werkzeugmaschinenbau geht es beispielsweise um appbasierte Steuerungs- und Bediensysteme für Bearbeitungszentren sowie um digitale Zwillinge und Plattformen für Condition Monitoring und vorausschauende Wartung von Zerspan- und Umformtechnik. Alles in allem wurde aus einem anfänglich marketinggetriebenen Thema ein strategisches Umsetzungsthema mit Substanz.

Speziell der Megatrend Plattformökonomie wird aus der Anbieterperspektive immer bedeutender. Große Plattformanbieter wie die bekannten IT-Giganten und Technologiekonzerne dringen seit geraumer Zeit ins industrielle Umfeld und in angestammte Maschinenbaumärkte vor. Es besteht die Gefahr, dass die direkte Kundenschnittstelle an branchenfremde Anbieter verloren geht und der Maschinenbau in die Rolle des Hardwarelieferanten abgedrängt wird. Mittelfristig werden sich nur die Konzepte durchsetzen, die den gesamten Wertstrom des Kunden und nicht nur einzelne Maschinen im Blick haben. Dies bietet neue Chancen, aber auch große Risiken für die Maschinenbaubranche. Denn noch ist nicht entschieden, wer sich die Innovationsführerschaft in dieser neuen Automatisierungssphäre erkämpfen und die Kundenschnittstelle besetzen kann. Die digitalen Plattformen, die sich am Markt durchsetzen, werden nicht nur von ihrer dominanten Marktposition profitieren. Vielmehr werden sie zum zentralen Knowhow-Träger zukünftiger digitaler Automatisierungskonzepte. Nicht wenige in der Branche befürchten, dass die jetzige Innovationsführerschaft und die Kundennähe verloren gehen, wenn es dem Maschinen- und Anlagenbau nicht gelingt, diese neue Sphäre zu beherrschen oder zumindest wesentlich daran beteiligt zu werden. Ziel für Maschinenbauunternehmen muss es daher sein, die direkte Kundenschnittstelle weiterhin zu kontrollieren und nicht an branchenfremde Anbieter - Internet-Giganten oder Technologiekonzerne - zu verlieren.

Dieser Wettstreit um die Innovationsführerschaft bei digitalen Lösungen und IIoTPlattformen ist entscheidend, aber noch nicht entschieden. Um seine starke Rolle bei Innovationen und beim Kundenzugang zu behalten, muss der Maschinenbau sich in der Plattformökonomie strategisch aufstellen und Unternehmen des Maschinenbaus sollten stärker zusammenarbeiten. Gerade unter den digitalen Plattformen werden nur wenige die Standards bzw. den Rahmen im industriellen Bereich setzen. Für den deutschen Maschinenbau wäre es wünschenswert, wenn nicht sogar überlebenswichtig, wenn einige erfolgreiche unter ihnen aus den eigenen Reihen kämen.

\subsection{Digitalisierung in der Anwenderperspektive}

In vielen Maschinenbauunternehmen wird Digitalisierung in erster Linie in der bisher beschriebenen Anbieterperspektive betrachtet. Bei den Kunden sollen durch Digitalisierung und KI weitere Effizienz und Rationalisierungspotenziale erschlossen werden. Dagegen wird der digitale Wandel in der Anwendersicht des Maschinenbaus bei den internen Unternehmensprozessen - von der Entwicklung, dem Produktmanagement, dem Einkauf über die Produktion bis zum Rechnungswesen, Vertrieb und Service - eher als schleichende Umsetzung wahrgenommen. Nichtsdestotrotz ist 
diese dritte Säule von Digitalisierungsstrategien - häufig als Industrie 4.0 bezeichnet ein wichtiger Faktor für die zukünftige Wettbewerbsfähigkeit von Maschinenbauunternehmen. Durch die Digitalisierung der internen Unternehmensprozesse ergeben sich vielfältige Möglichkeiten zur Prozessoptimierung. Zu unterscheiden ist zwischen der Implementierung einzelner digitaler Technologien im Unternehmen und der umfassenden Vernetzung des Unternehmens durch erweiterte Software-Systeme bzw. digitale Steuerungssysteme (Abb. 2).

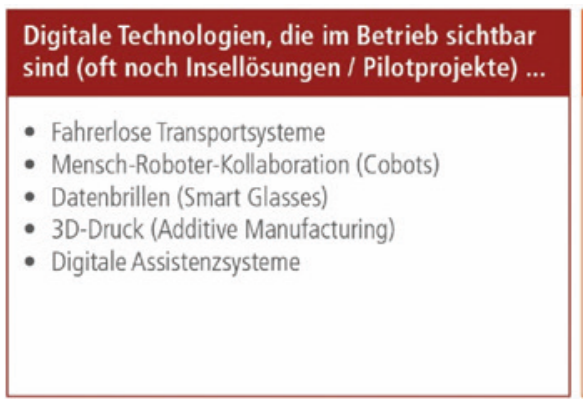

... und Software-Systeme als verborgene
Elemente der digitalen Transformation
- ERP, MES mit erweiterten Funktionalitäten und
mit Echtzeit-Analyse
- PLM, CAx (CAD/CAM), Digitaler Zwilling
(Simulation)
- Software-Bots (RPA - Robotic Process
Automation)
- Künstliche Intelligenz und autonome
Software-Systeme

Abb. 2. Digitalisierung der internen Prozesse im Maschinenbau. (Eigene Darstellung nach IMU Institut)

Zwischen den digitalen Technologien und den Software-Systemen besteht ein großer, direkt wahrnehmbarer Unterschied. Digitale Technologien wie fahrerlose Transportsysteme, Cobots, Datenbrillen und 3D-Druck sind für die Menschen im Betrieb sichtbar; sie werden zunächst meist als Pilotprojekt oder Insellösung implementiert. Damit sind diese digitalen Technologien erfahrbar und rücken ins Bewusstsein der betrieblichen Akteure. Die Unmenge an Daten, die dadurch erzeugt wird (Big Data), ist jedoch nicht sichtbar. Die Erfassung und Auswertung großer Datenmengen erzeugt Handlungsbedarfe beim betrieblichen Datenschutz und bei Themen wie personenbezogener Datenauswertung sowie Leistungs- und Verhaltenskontrolle. Bei den digitalen Technologien, die im betrieblichen Alltag auf dem Shopfloor sichtbar sind, lassen sich diese Handlungsbedarfe materiell festmachen.

$\mathrm{Zu}$ den Kernpunkten der Digitalisierung in Anwenderperspektive von Industrieunternehmen gehört die umfassende Vernetzung der Unternehmensprozesse im Sinne von Cyber-Physical-Systems (CPS). Mit CPS als Kernelement von Industrie 4.0 soll eine Durchgängigkeit in der Prozesskette von der Bestellung/Entwicklung bis zu Auslieferung/Service (End-to-End) erreicht und die echtzeitdatenbasierte Analyse und Optimierung von Produktionsprozessen ermöglicht werden. Damit werden in den Betrieben Ziele verfolgt wie: größere Effizienz, höhere Flexibilität, bessere Qualität und kürzere Produkteinführungszeit (Time-to-Market). Mit digitaler Vernetzung durch Visualisierung und Transparenz über alle Prozesse sowie Echtzeitfähigkeit sollen diese Ziele erreicht werden.

Ein immer wichtiger werdendes Element der digitalen Transformation ist der digitale Zwilling als softwarebasiertes, virtuelles Abbild des Produkts, der Produktion 
und der Prozessperformance. Ein solcher digitaler Zwilling kann die Basis bilden für eine kürzere Produkteinführungszeit, für eine virtuelle Inbetriebnahme und damit eine signifikant verkürzte reale Inbetriebnahme von Anlagen, für vorausschauende Wartung und höhere Verfügbarkeit, für minimierte Rüstprozesse und höhere Maschinenlaufzeiten, für größere Transparenz im Produktionsprozess und Vermeidung von Verschwendung (im Sinne von Lean Management) sowie für digitale Services und neue Geschäftsmodelle. Bisher hat die deutsche Industrie wie auch der Maschinenbau in der realen Produktionswelt ihre Exzellenz unter Beweis gestellt und eine Spitzenstellung erreicht. In der Zukunft wird es laut befragten Experten darum gehen, die Verbindung von realer und digitaler Welt wie auch von menschlicher und künstlicher Intelligenz zu schaffen und damit den technologischen Vorsprung zu halten.

Alles in allem ist die übergreifende Vernetzung mittels Software-Systemen im Betrieb und in der Wertschöpfungskette ein nicht sichtbares, verborgenes Element der digitalen Transformation, das für Beschäftigte und Betriebsräte schwerer zu greifen ist. Umso mehr sollte auch hier ein Hauptaugenmerk der Mitbestimmungsträger auf Themen wie Arbeitsgestaltung und Datenschutz gelegt werden. Zumal der Blick auf diesen Kernbereich der digitalen Transformation häufig durch die sichtbaren digitalen Technologien verdeckt wird, mit denen im Betrieb experimentiert wird und die als Pilotprojekte implementiert werden.

\subsection{Beteiligung von Beschäftigten und Betriebsräten}

Die Beteiligung von Betriebsräten und Beschäftigten - die vierte Säule von Digitalisierungsstrategien - ist für den Erfolg der digitalen Transformation entscheidend. Wie von den befragten Experten immer wieder betont wurde, ist die digitale Transformation keine rein technische Angelegenheit. Neben digitalen Technologien und Software-Systemen als technischen Befähigern (,Technical Enablers“) sind für die Umsetzung der internen Digitalisierung die „Non-technical Enablers“ wie Change Management, Unternehmenskultur und das Aufsetzen auf Lean-Erfahrungen entscheidend.

Ein ganzheitlicher Gestaltungsansatz und ein Digitalisierungsverständnis sind erforderlich, die gleichermaßen die technologischen, organisatorischen und arbeitsbezogenen Dimensionen eines Unternehmensprozesses mit ihren engen Wechselwirkungen in den Blick nehmen und diesen als sozio-technisches System begreifen (Hirsch-Kreinsen et al. 2018). Von der Prämisse ausgehend, dass Digitalisierung gestaltbar ist, ergibt sich ein „Handlungsauftrag für Interessenvertretungen, diese Entwicklung nach Kräften zu beeinflussen und zu prägen, um die Chancen für die Beschäftigten so gut wie möglich zu verbessern, sei es mit Blick auf die Handlungsautonomie oder mit Blick auf Qualifizierungs- und Entwicklungspotenziale“ (Falkenberg et al. 2020: 14). Auch IMU-Studien mit zahlreichen Expertengesprächen im Maschinenbau und anderen Branchen bestätigen, dass die digitale Transformation ohne eine beteiligungsorientierte und partizipative Unternehmenskultur kaum erfolgreich gestaltet werden kann (Dispan, Schwarz-Kocher 2018; Dispan 2020). Zum erforderlichen Change Management gehört auch, dass Betriebsräte von Beginn an eingebunden sind und dass Mitarbeiter vorbereitet und befähigt werden, um mit den Anforderungen der Digitalisierung in Zukunft umgehen zu können. 


\section{Beschäftigungswandel in der digitalen Transformation}

Mit der digitalen Transformation wird es im Maschinenbau wie auch in der Industrie insgesamt einen Wandel der Beschäftigung in allen betrieblichen Bereichen und Funktionen geben. Es kommt zu strukturellen Veränderungen zwischen unterschiedlichen Beschäftigtengruppen wie auch zu qualitativen Veränderungen der Arbeitsbedingungen. Quantitative Arbeitsplatzeffekte durch Digitalisierung werden im Maschinenbau durch gegenläufige Prozesse geprägt sein: Neue digitale Angebote und damit erreichbares Wachstum werden Arbeitsplätze sichern und schaffen. Dagegen werden die Effizienzgewinne durch Digitalisierung bei den internen Prozessen Arbeitsplätze verändern oder gar überflüssig machen. Unter der Prämisse „Wachstum durch digitale Angebote“ wird der Saldo aus beidem in den nächsten Jahren neutral bis eher positiv sein. Mittel- bis langfristig gesehen wird es im Maschinenbau aufgrund der Rationalisierungseffekte eher zu einem Arbeitsplatzabbau kommen. Noch stärker als direkte Bereiche in der Produktion werden dann die klassischen Büro- und Informationstätigkeiten unter Druck kommen. Digitale Tools, Software-Bots ${ }^{3}$ und die durchgängige Vernetzung greifen insbesondere bei Tätigkeiten entlang der ,,indirekten Kette" vom Vertrieb über Entwicklung, Konstruktion, Einkauf, Produktionsplanung/steuerung, Buchhaltung, Controlling bis hin zu Aftersales.

Mit dem Einsatz von digitalen Technologien und der zunehmenden Vernetzung der Unternehmensprozesse im Maschinenbau verändern sich die Arbeitsbedingungen für die Beschäftigten in Produktion, Büros, Außentätigkeiten etc. In manchen Bereichen sind die Veränderungen heute bereits spürbar, in anderen Bereichen werden mit zunehmender Digitalisierung künftig starke Veränderungen erwartet. Was sich heute bereits in Maschinenbauunternehmen abzeichnet und zukünftig wohl herausbildet, ist sehr differenziert für die einzelnen Teilbranchen und auch Tätigkeitsbereiche zu betrachten. „Eindeutige“ Entwicklungsstränge konnten aus den Betriebsfallstudien nicht abgeleitet werden. Somit wird im Folgenden schlaglichtartig auf einige betriebliche Trends eingegangen.

\subsection{Veränderungen bei Produktionstätigkeiten}

Ganz unterschiedliche Effekte sind innerhalb eines Unternehmens aus dem Werkzeugmaschinenbau in zwei Produktionsbereichen zu beobachten, in denen Industrie 4.0 im Hinblick auf die gesamte Prozesskette bereits frühzeitig umgesetzt wurde. Im Produktionsbereich A wird von einem klaren „Upgrading“ für die Werker berichtet. Die Mitarbeiter sind mit Handheld-Geräten ausgestattet und verfügen damit über alle arbeitsrelevanten Informationen in Echtzeit. Die papierlose Produktion wird durch digitales Shopfloor Management unterstützt. Die komplette Prozesskette von der Konstruktion bis zur Auslieferung wurde durchgängig vernetzt. Durch die Digitalisierung ist eine hohe Transparenz in die Prozesse in allen Bereichen

3 Mit Software-Bots bzw. Robotic Process Automation (RPA) können zahlreiche Bürotätigkeiten, insbesondere Routinearbeiten, automatisiert werden (Stroheker 2020). 
gekommen. Für die direkten Mitarbeiter gab es eine Anreicherung bei den Arbeitsinhalten sowie eine ausgeprägtere Verantwortlichkeit für den Produktionsprozess und die Qualität. Für das reibungslose Funktionieren dieser „digitalen Produktion“ muss das Prozessverständnis jedes Mitarbeiters deutlich größer als zuvor sein. Die Mitarbeiter benötigen höheres Prozess-Knowhow. Wo zuvor Produktionssteuerer bzw. Disponenten die Aufträge durchgesteuert haben, steuert heute ein MES als Feinplanungstool. „Die Mitarbeiter müssen jetzt eigenständig wissen, was muss ich tun, was passiert vor mir, was nach mir" (Exp). In diesem Produktionsbereich A haben die nicht mehr benötigten Disponenten neue Aufgaben bekommen und die Mitarbeiterzahl ist insgesamt gleich geblieben.

Ein anderes Bild bei Industrie 4.0-Effekten zeigt sich im Produktionsbereich B desselben Werkzeugmaschinenbauers. Dort wurde die Belegschaft durch digitale Workflows im End-to-End-Prozess und durch digitale Möglichkeiten der Mehrmaschinenbedienung deutlich von rund 100 auf 70 Beschäftigte reduziert. Gleichwohl konnten im prosperierenden Unternehmen den in diesem Bereich nicht mehr benötigten Mitarbeitern durch Qualifizierung neue Perspektiven eröffnet werden. Der Beschäftigungsabbau in diesem Produktionsbereich betraf ganz unterschiedliche Funktionen: Entfallen sind beispielsweise Mitarbeiter in Prozesssteuerung, Arbeitsvorbereitung und Vertrieb durch hochautomatisierte Prozesse in indirekten Bereichen - von der konfigurierten Bestellung bis hin zum ersten Produktionsschritt. Auch in der Fertigung wurde eine vernetzte Produktions-U-Linie mit integrierten Robotern eingeführt. Infolge der Mehrmaschinenbedienung und der mannlosen Nacht- und Wochenendschichten werden dort heute nur noch drei Facharbeiter in zwei Schichten benötigt, statt zuvor zehn Werker in zwei Schichten bei Einzelmaschinenbedienung. Von den überwiegend jungen Facharbeitern werden eine große Leistungsbereitschaft und Flexibilität bei der Arbeit eingefordert. Leistungsverdichtung sei aber bisher nicht unmittelbar als Digitalisierungsfolge spürbar, so ein befragter Betriebsrat.

Die Flexibilisierung der Arbeit im Maschinenbau wird sich durch die Möglichkeiten der Digitalisierung weiter forcieren. Bislang wird die Flexibilisierung der Arbeitszeit und des Arbeitsorts vor allem bei Beschäftigtengruppen aus Angestelltenbereichen umgesetzt. Digitale Technologien ermöglichen eine Ausweitung dieser Formen der Flexibilisierung auf Beschäftigte im direkten Bereich in unterschiedlicher Intensität und Reichweite, zum Beispiel auf Instandhalter, aber auch auf Maschinenbediener und auf Montagefachkräfte. Insgesamt wird für Produktionstätigkeiten ein Wandel von stärker mechanischer Arbeit hin zu mehr Steuerungs-, Kontroll- und Überwachungsfunktionen erwartet.

\subsection{Veränderungen im Service}

Die Arbeitsbedingungen in Service-Bereichen des Maschinenbaus könnten sich infolge der digitalen Transformation in den nächsten Jahren stark verändern, wie am Beispiel der beiden Teilbranchen Aufzüge/Fahrtreppen und Fördertechnik/ Intralogistik gezeigt wird. Durch digitale Wartungssteuerung, mobile Endgeräte und weitere digitale Tools und Technologien könnten sich für viele Servicemonteure im Außeneinsatz die Wartungsrouten und Arbeitsinhalte, aber auch die 
Eigenverantwortung, Selbststeuerung und Selbstorganisation deutlich verändern. Ein weiterer Effekt der digitalen Wartungssteuerung in Kombination mit den Möglichkeiten der digitalen Plattformen (wie vorausschauende Wartung) und digitalen Assistenzsysteme (Ferndiagnose, Teleservice, Remote Support) könnte sein, dass es zu einer Aufgliederung bzw. Ausdifferenzierung bei den Servicemonteuren kommt. Betriebsräte aus der Branche befürchten, dass einer „Aufspreizung Tür und Tor geöffnet wird“ bzw. dass der ,Weg zur Klassengesellschaft bei den Servicemonteuren" beschritten wird. Während bei den Meistern durch automatisierte Auftragssteuerung ihr Part des Disponierens zwar entfallen würde, sie aber nach wie vor eine Entscheider- und Schnittstellenfunktion in den Serviceniederlassungen einnehmen werden, könnte es bei den Servicemonteuren zu einer starken Differenzierung in drei „Klassen von Servicemonteuren“ mit unterschiedlichen Qualifikationen und Entgeltbedingungen kommen:

- Technische Experten mit umfangreicher technischer Ausbildung (mechanisch, elektronisch, digital) für komplizierte Reparatur- und Wartungsaufgaben

- Standardmonteure mit Facharbeiterausbildung für Reparaturen und Wartung, die auch die Rufbereitschaft mit abdecken

- Einfache Monteure, die auf Basis einer Schulung für Ölwechsel und einfache Routinetätigkeiten eingesetzt werden

\subsection{Leistungsverdichtung}

Ein weiterer Aspekt, der mit der digitalen Transformation einhergeht, sind Änderungen der Arbeitsbelastungen im Maschinenbau. Die Automatisierung von Routinetätigkeiten durch Software-Bots oder andere digitale Tools wird von den Beschäftigten zwiespältig erlebt. Zwischen Entlastung und Belastung liegt ein Spannungsfeld: Einerseits wird es als Vorteil empfunden, dass oftmals „lästige“ Arbeiten wegfallen und die Konzentration auf wesentliche oder strategische Aufgaben gelenkt werden kann. Andererseits entfallen dadurch leichte, entlastende Tätigkeiten, die der Erholung zwischen Phasen anstrengenden oder hochkonzentrierten Arbeitens dienen. Damit kann die Digitalisierung zu einer Leistungsverdichtung führen. Zudem gibt der Wegfall von Tätigkeiten aus Sicht von Betriebsräten häufig Anlass zu Diskussionen um die Zahl der Beschäftigten und selten Anlass für eine Verbesserung der Tätigkeitszuschnitte.

Eine Belastung für Mitarbeiter vor allem in den größeren Maschinenbauunternehmen stellt die Vielzahl der zu nutzenden Software-Programme und die Vielfalt von Informationen aus unterschiedlichen Kanälen dar, mit denen die Beschäftigten umgehen müssen. Durch ,überbordende Kommunikation“ und die „Informationsflut“, der viele Beschäftigte ausgesetzt sind, werden nicht zuletzt auch Produktivitätseffekte, die aus der Digitalisierung und Vernetzung erzielt werden, wieder aufgezehrt oder schlagen ins Gegenteil um.

Datenschutz, der Schutz personenbezogener Daten wurde in den Betriebsfallstudien besonders von Seiten der Betriebsräte als wichtiges Thema ins Feld geführt. Besonders kritisch wird der durch digitale Technologien und Software-Systeme ermöglichte „gläserne Mitarbeiter" gesehen. Aus mehreren Betriebsfallstudien 
berichten befragte Experten, dass durch die digitale Vernetzung der Prozesse eine volle Transparenz in vielen Bereichen von Maschinenbauunternehmen erreicht wurde. Die Produktionsmitarbeiter loggen sich am Touchscreen-Monitor, am HandheldGerät oder anderswo ein und hinterlassen ihre Datenspur. In einem der Fallbetriebe werden die erfassten Daten zu Stillständen, Störungsmeldungen, Fehlerkennung auch personenbezogen für einen begrenzten Zeitraum gespeichert und sind von Führungskräften einsehbar. Zwar wird in diesem Betrieb Leistungs- und Verhaltenskontrolle durch eine Betriebsvereinbarung ausgeschlossen; de facto werde der Mitarbeiter aber bei einem Störfall gleich zum Sündenbock gemacht und ,es wird Druck auf ihn ausgeübt, ohne dass eine ehrliche Fehleranalyse durchgeführt wird“ (Exp). Alles in allem gilt es für die Betriebsräte nach wie vor, so weit wie möglich personenbezogene Auswertungen zu verhindern und Leistungs- und Verhaltenskontrolle auszuschließen.

\subsection{Kompetenzanforderungen und Qualifikationen}

Die digitale Transformation stellt die Beschäftigten vor vielfältige neue Anforderungen. Bei den Betriebsfallstudien im Maschinenbau und den Experteninterviews wurden zwei Bereiche hervorgehoben: „Kompetenzanforderungen und Qualifikationen“, auf die im Folgenden eingegangen wird, sowie ,agile Arbeitsformen“ als neue Anforderung der Arbeitsorganisation.

IT- und Software-Kompetenzen als Anforderung für den digitalen Wandel von Unternehmen liegen auf der Hand, allein schon weil die Produkte des Maschinenbaus wie auch die internen Prozesse immer stärker digitalisiert werden. Das gilt in der Breite, weil fast alle Beschäftigten Software anwenden und sich fortlaufend in neue Programme, neue Apps und Tools einarbeiten sollen. Gleichzeitig gilt das in der Tiefe, weil der Bedarf an IT-Spezialisten und Software-Ingenieuren im Maschinenbau immer größer wird. Dies wird von einer VDMA-Studie bestätigt: Die größten Problemfelder für Maschinenbauer bei der Digitalisierung von Produkten (Entwicklung von Software, IT-Hardware oder Automatisierungstechnik) liegen im Bereich der „Human Resources“ - so ein Ergebnis der VDMA-Studie (Oetter 2018). Mit Abstand größtes Problemfeld für die Digitalisierung ist im Jahr 2018 demnach die Personalverfügbarkeit; es folgen der Knowhow-/Technologietransfer sowie die Ausund Weiterbildung der Mitarbeiter.

Aus den Betriebsfallstudien lassen sich zwei mögliche Entwicklungsstränge für die Kompetenzanforderungen und Qualifikationen in den direkten Bereichen des Maschinenbaus ableiten. Es bleibt offen, ob für Produktionsarbeit im digitalisierten Maschinenbau höhere Qualifikationen oder geringere Qualifikationen der Mitarbeiter erforderlich sind. Das Spektrum der Einschätzungen aus den Experteninterviews passt zu zwei der drei „Entwicklungsszenarien zur Zukunft digitaler Arbeit“", wie sie in der Studie „Digitalisierter Maschinenbau“ von der IG Metall veröffentlicht wurden (Hirsch-Kreinsen 2017). Demnach könnte es bei der Produktionsarbeit im Maschinenbau zu einem „Upgrading-Szenario“ oder zu einem „PolarisierungsSzenario“ kommen. Das dritte Szenario „Automatisierte Fabrik“ scheint eher für die Anwenderbranchen des Maschinenbaus und nicht für den Maschinenbau selbst relevant zu sein. Die zwei aus den Experteninterviews abgeleiteten widersprüchlichen 
Entwicklungsstränge für künftige Qualifikationserfordernisse in den direkten Bereichen des Maschinenbaus sind demnach:

- „Upgrading von Arbeit“ (mit steigenden Qualifikationen): Wird es durch höhere Anforderungen durch Digitalisierung der internen Prozesse und erforderlichem umfassenden Prozess-Knowhow eine Aufwertung bei den FacharbeiterQualifikationen im Maschinenbau geben? Dafür sprechen die große Varianz bei den Produkten und Losgröße-1-Erfordernisse in vielen Bereichen des Maschinenbaus, die eine hohe Flexibilität und Genauigkeit in der Produktion von Maschinen in einer von Software-Systemen und digitalen Technologien geprägten Prozesslandschaft erfordern.

- „Polarisierung von Arbeit“" (mit „Gewinnern“ und „Verlierern“ bei den Qualifikationen):

- Wird es durch digitale Assistenzsysteme und autonome Software-Systeme eine Abwertung bei den Qualifikationen geben? Geht der Weg von der Facharbeiter-Dominanz im Maschinenbau hin zum verstärkten Einsatz Angelernter in der Produktion? Dafür sprechen enge Arbeitsanweisungen durch digitale Assistenzsysteme sowie standardisierte und „verriegelte“ Montageprozesse, die zu einfacheren operativen Tätigkeiten im Maschinenbau und damit zu einer Dequalifizierung führen können.

- Wird es auf der anderen Seite mehr anspruchsvolle hochqualifizierte Tätigkeiten für Experten zur Wartung und Installation der Systeme geben? Dafür sprechen komplexere Tätigkeiten mit hohen Qualifikationsanforderungen für eine ,kleine Facharbeiterelite“ im Maschinenbau, wogegen die bisherigen mittleren Qualifikationsgruppen mit sinkenden Anforderungsniveaus konfrontiert werden. Kommt es also zu einer Gleichzeitigkeit von Upgrading auf der einen und Dequalifizierung auf der anderen Seite?

Laut den Betriebsfallstudien zeichnet sich in den Produktionsbereichen des Maschinenbaus eher eine Polarisierung bei den Qualifikationen ab. Für die Mehrzahl der befragten Experten wird sich die Schere zwischen „Gewinnern“ und „Verlierern“ bei den Qualifikationen in den direkten Bereichen des digitalisierten Maschinenbaus künftig öffnen.

Dagegen könnte es in den indirekten Bereichen zu einer Entwicklung kommen, die eher dem „Automatisierungsszenario“ entspricht. Durch Software-Systeme und Bots werden sich nicht nur die Arbeitszuschnitte in den Bürobereichen verändern, sondern es wird zu einer umfassenden Automatisierung von Bürotätigkeiten kommen. In der Konsequenz könnte dies zu einem rationalisierungsbedingten Arbeitsplatzabbau führen, der fast alle Qualifikationsstufen trifft - mit Ausnahme höherer Führungsebenen und hochqualifizierter Experten.

Gleichwohl ist für Beschäftigte in allen Bereichen des Maschinenbaus festzuhalten, dass an Qualifizierung für die neuen Anforderungen und die neuen digitalen Technologien kein Weg vorbeiführt. Der digitalen Transformation müssen sich alle Beschäftigten durch Weiterbildung stellen. Beim Thema Qualifikationen kommt selbstverständlich auch der betrieblichen Ausbildung erhebliches Gewicht zu. Bei einigen der Betriebsfallstudien werden die Herausforderungen durch Industrie 4.0 
und Digitalisierung in eigene Ausbildungskonzepte umgesetzt. So wurde beispielsweise bei einem Werkzeugmaschinenhersteller ein Smart Education Center innerhalb der Werkhalle aufgebaut, in dem Auszubildende an wichtige Digitalisierungsthemen praktisch herangeführt werden und wo ihnen das Gesamtsystem Maschinenbau nahegebracht wird. Insgesamt vollzieht sich in den Maschinenbauunternehmen ein Wandel von der klassischen mechanischen Ausbildung hin zu mehr IT- und Steuerungskenntnissen. Seit Mitte 2018 sind bei den industriellen Metall- und Elektroberufen Themen wie Digitalisierung der Arbeit, Datenschutz und Informationssicherheit fester Bestandteil der Ausbildung geworden. Generell sind die Berufsbilder in der Metall- und Elektroindustrie prozessorientiert und auf die von Industrie 4.0 geforderte Systemorientierung sowie der damit verbundenen Wertschöpfung und Vernetzung gerichtet.

\section{Fazit: Gestaltung guter Arbeit und Beteiligung}

Die digitale Transformation gehört zu den wichtigsten Entwicklungstrends für die Industrie im Allgemeinen und für den Maschinenbau als Industrieausrüster im Speziellen. Kein Maschinenbauunternehmen darf sich der digitalen Transformation verschließen, wenn es nicht seine Zukunftsfähigkeit verspielen will. Einige vor allem größere Unternehmen verfolgen bereits eine Digitalisierungsstrategie, für die restlichen spielen zumindest Elemente der digitalen Transformation in ihren Produkten, Prozessen oder auch Geschäftsmodellen eine Rolle. Da die Digitalisierung über kurz oder lang die Beschäftigung und die Arbeitsbedingungen in fast allen betrieblichen Tätigkeitsfeldern verändert, gibt es umfassende Handlungsbedarfe für die Interessenvertretung. Auf arbeitspolitische Handlungsfelder und Gestaltungsmöglichkeiten der betrieblichen Mitbestimmung unter der zentralen Prämisse „,mitbestimmte Einführungsprozesse“ geht ein Forschungsreport der Hans-Böckler-Stiftung ein (Falkenberg et al. 2020: 18-24). Laut der IMU-Studie „Digitalisierung im Maschinenbau“ sind folgende Gestaltungsfelder für die Interessenvertretung hervorzuheben (Dispan, Schwarz-Kocher 2018: 72-84):

- Betriebsrats-Strategie für die digitale Transformation erarbeiten

- Gute Arbeit im digitalisierten Maschinenbau gestalten

- Prozessorientierte Betriebsvereinbarung als Rahmen für die Digitalisierung abschließen

- Beteiligungsprozesse für die Beschäftigten organisieren

Der tiefgreifende Wandel der Arbeitswelt durch die digitale Transformation erfordert die umfassende Beteiligung der Mitbestimmungsträger in den Unternehmen. Insbesondere geht es um die frühzeitige Einbindung von Betriebsräten, um die (Mit-) Gestaltung von Digitalisierungsprozessen und um Regelungsbedarfe bei Fragen der Arbeitsgestaltung, der Arbeitsbedingungen, der Personalentwicklung und des Datenschutzes. Betriebsräte sollten sich aber nicht auf Regelungen zum Datenschutz der Beschäftigten beschränken, sondern das gesamte Spektrum der arbeits- und betriebspolitischen Themen in den Blick nehmen, weil Digitalisierung ein Querschnittsthema mit vielfältigen Auswirkungen auf die Arbeitswelt ist. 
Diese breite Palette an Gestaltungsfeldern zeigt, wie hoch die Anforderungen an Betriebsräte sind: Die digitale Transformation ist mit einer hohen Komplexität und mit kaum vorhersehbaren Prozessen verbunden. Manches im Bereich der SoftwareSysteme und digitalen Zwillinge läuft eben ,eher im Verborgenen“ ab, mit „unsichtbaren" Veränderungen, deren Wirkungen auf Arbeit schwer zu erkennen sind. Vielfach stoßen Betriebsräte hierbei wegen begrenzter Ressourcen und mangelnder Qualifizierung an ihre Grenzen. Damit die digitale Transformation zum Erfolgsprojekt für die Branche und die Beschäftigten wird, gilt es daher, die Betriebsräte hinsichtlich Qualifizierung und Ressourcenausstattung zu stärken.

Eine zentrale Aufgabe der Mitbestimmungsträger im Maschinenbau ist es, Beteiligungsprozesse für die Beschäftigten $\mathrm{zu}$ organisieren und in den betrieblichen Abläufen zu verankern. Durch Beteiligung der Beschäftigten gelingt es dem Betriebsrat, Themen zu setzen und gute Arbeitsbedingungen zu erreichen. Nach wie vor bleibt die Gestaltung guter Arbeit eines der wichtigsten Handlungsfelder für Mitbestimmungsträger. Insgesamt sollten sichere Arbeitsplätze und gute Arbeitsbedingungen in der gesamten Branche das Ziel sein.

\section{Literatur}

Dispan, J.: Branchenanalyse Medizintechnik. Beschäftigungs-, Markt- und Innovationstrends. Düsseldorf (= Working Paper der Hans-Böckler-Stiftung, Nr. 183/2020) (2020)

Dispan, J., Schwarz-Kocher, M.: Digitalisierung im Maschinenbau. Entwicklungstrends, Herausforderungen, Beschäftigungswirkungen, Gestaltungsfelder im Maschinen- und Anlagenbau. Düsseldorf (= Working Paper der Hans-Böckler-Stiftung, Nr. 94/2018) (2018)

Falkenberg, J., Haipeter, T., Krzywdzinski, M., Kuhlmann, M., Schietinger, M., Virgillito, A.: Digitalisierung in Industriebetrieben. Düsseldorf (= Report der Hans-Böckler-Stiftung, Nr. 6/2020) (2020)

Hirsch-Kreinsen, H.: Digitalisierter Maschinenbau - Wandel und Entwicklungschancen qualifizierter Arbeit. IG Metall, Frankfurt a. M. (2017)

Hirsch-Kreinsen, H., Ittermann, P., Niehaus, J. (Hrsg.): Digitalisierung industrieller Arbeit. Die Vision Industrie 4.0 und ihre sozialen Herausforderungen, 2. Aufl. Nomos, Baden-Baden (2018)

Kuhlmann, M., Voskamp, U.: Digitalisierung und Arbeit im niedersächsischen Maschinenbau. Göttingen (SOFI-Arbeitspapier 2019-15) (2019)

Oetter, C.: Presse Preview AMB 2018 (VDMA). Stuttgart (2018)

Pfeiffer, S., Lee, H., Zirnig, C., Suphan, A.: Industrie 4.0 - Qualifizierung 2025. VDMA, Frankfurt (2016)

Stroheker, S.: Buchung wie von Geisterhand. Comput. Arbeit. 1, 20-24 (2020)

VDMA - Verband Deutscher Maschinen- und Anlagenbau: Industrie 4.0 - Bausteine bereiten den Weg. VDMA, Frankfurt a. M. (2019) 
Open Access Dieses Kapitel wird unter der Creative Commons Namensnennung 4.0 International Lizenz (http://creativecommons.org/licenses/by/4.0/deed.de) veröffentlicht, welche die Nutzung, Vervielfältigung, Bearbeitung, Verbreitung und Wiedergabe in jeglichem Medium und Format erlaubt, sofern Sie den/die ursprünglichen Autor(en) und die Quelle ordnungsgemäß nennen, einen Link zur Creative Commons Lizenz beifügen und angeben, ob Änderungen vorgenommen wurden.

Die in diesem Kapitel enthaltenen Bilder und sonstiges Drittmaterial unterliegen ebenfalls der genannten Creative Commons Lizenz, sofern sich aus der Abbildungslegende nichts anderes ergibt. Sofern das betreffende Material nicht unter der genannten Creative Commons Lizenz steht und die betreffende Handlung nicht nach gesetzlichen Vorschriften erlaubt ist, ist für die oben aufgeführten Weiterverwendungen des Materials die Einwilligung des jeweiligen Rechteinhabers einzuholen. 